Algebraic $8 \mathcal{G}$ Geometric $\mathcal{T}_{\text {opology }}$

Volume 4 (2004) 399-406

Published: 10 June 2004

ATG

\title{
Bounds for the Thurston-Bennequin number from Floer homology
}

\author{
Olga Plamenevskaya
}

\begin{abstract}
Using a knot concordance invariant from the Heegaard Floer theory of Ozsváth and Szabó, we obtain new bounds for the ThurstonBennequin and rotation numbers of Legendrian knots in $S^{3}$. We also apply these bounds to calculate the knot concordance invariant for certain knots.
\end{abstract}

AMS Classification 57R17, 57M27

Keywords Legendrian knot, Thurston-Bennequin number, Heegaard Floer homology

\section{Introduction}

Let $K$ be a Legendrian knot of genus $g$ in the standard tight contact structure $\xi_{\text {standard }}$ on $S^{3}$. It is well-known that the Thurston-Bennequin and rotation numbers of $K$ satisfy the Thurston-Bennequin inequality

$$
t b(K)+|r(K)| \leq 2 g-1 .
$$

Although sharp in some cases (e.g. right-handed torus knots), in general this bound is far from optimal. Better bounds can be obtained using Kauffman and HOMFLY polynomials [FT], Ta]. The Kauffman polynomial bounds are easily seen to be sharp for left-handed torus knots; they also allow one to determine the values of the maximal Thurston-Bennequin number for all two-bridge knots $\mathrm{Ng}$.

In this paper we use the Ozsváth-Szabó knot concordance invariant $\tau(K)$ introduced in [OS5], Ra to establish a new bound for the Thurston-Bennequin and the rotation number of a Legendrian knot. We have

Theorem 1 For a Legendrian knot $K$ in $\left(S^{3}, \xi_{\text {standard }}\right)$

$$
t b(K)+|r(K)| \leq 2 \tau(K)-1 .
$$


For a large class of knots ("perfect" knots Ra ), $\tau(K)=-\sigma(K) / 2$, where $\sigma(K)$ is the signature of the knot (with the sign conventions such that the right-handed trefoil has signature -2). All alternating knots are perfect [OS4, which gives

Corollary 1 If $K \subset\left(S^{3}, \xi_{\text {standard }}\right)$ is an alternating Legendrian knot, then

$$
t b(K)+|r(K)| \leq-\sigma(K)-1 .
$$

In particular, for alternating knots with $\sigma(K)>0$, the Thurston-Bennequin inequality is not sharp, and $t b(K)$ can never be positive.

Note that this bound is usually not sharp even for two-bridge knots and knots with few crossings (as can be seen from the calculations in $|\mathrm{Ng}|$ ).

It is shown in OS5 that $|\tau(K)| \leq g^{*}(K)$, where $g^{*}(K)$ is the four-ball genus of $K$. We therefore recover a bound due to Rudolph [Ru] :

Corollary $2 \quad t b(K)+|r(K)| \leq 2 g^{*}(K)-1$.

We prove Theorem 1 by examining the Heegaard Floer invariants of contact manifolds obtained by Legendrian surgery. The Heegaard Floer contact invariants were introduced by Ozsváth and Szabó in OS1; to an oriented contact 3 -manifold $(Y, \xi)$ with a co-oriented contact structure $\xi$ they associate an element $c(\xi)$ of the Heegaard Floer homology group $\widehat{H F}(-Y)$, defined up to sign. Conjecturally, the Heegaard Floer contact invariants are the same as the Seiberg-Witten invariants of contact structures constructed in [KM]. The definition of $c(\xi)$ uses an open book decomposition of the contact manifold; the reader is referred to OS1 for the details.

Acknowledgements I am grateful to Peter Kronheimer and Jake Rasmussen for illuminating discussions.

\section{The Invariant $\tau(K)$ and Surgery Cobordisms}

In this section we collect the relevant results of Ozsváth, Szabó, and Rasmussen.

For a knot $K \subset S^{3}$, the invariant $\tau(K)$ is defined via the Floer complex of the knot; we will need its interpretation in terms of surgery cobordisms OS5].

We use notation of [OS3]. Consider the Heegaard Floer group $\widehat{H F}(Y)$ of a 3 -manifold $Y$, and recall the decomposition $\widehat{H F}(Y)=\bigoplus_{\mathfrak{s} \in \operatorname{Spin}^{c}(Y)} \widehat{H F}(Y, \mathfrak{s})$. 
As described in OS2, a cobordism $W$ from $Y_{1}$ to $Y_{2}$ induces a map on Floer homology. More precisely, a $\operatorname{Spin}^{c} \operatorname{cobordism}(W, \mathfrak{s})$ gives a map

$$
\widehat{F}_{W, \mathfrak{s}}: \widehat{H F}\left(Y_{1}, \mathfrak{s} \mid Y_{1}\right) \rightarrow \widehat{H F}\left(Y_{2}, \mathfrak{s} \mid Y_{2}\right) \text {. }
$$

For a knot $K$ in $S^{3}$ and $n>0$, let $S_{-n}^{3}(K)$ be obtained by $-n$-surgery on $K$, and denote by $W$ the cobordism given by the two-handle attachment. The Spin $^{c}$ structures on $W$ can be identified with the integers as follows. Let $\Sigma$ be a Seifert surface for $K$; capping it off inside the attached two-handle, we obtain a closed surface $\widehat{\Sigma}$ in $W$. Let $\mathfrak{s}_{m}$ be the Spin ${ }^{c}$-structure on $W$ with $\left\langle c_{1}\left(\mathfrak{s}_{m}\right),[\widehat{\Sigma}]\right\rangle-n=2 m$. Accordingly, the $\operatorname{Spin}^{c}$ structures on $S_{-n}^{3}(K)$ are numbered by $[m] \in \mathbb{Z} / n \mathbb{Z}$. The cobordism $\left(W, \mathfrak{s}_{m}\right)$ induces a map from $\widehat{H F}\left(S^{3}\right)$ to $\widehat{H F}\left(S_{-n}^{3}(K),[m]\right)$; it will be convenient to think of $\left(W, \mathfrak{s}_{m}\right)$ as a cobordism from $\left(-S_{-n}^{3}(K),[m]\right)$ to $-S^{3}$, and consider the associated map $\widehat{F}_{n, m}: \widehat{H F}\left(-S_{-n}^{3}(K),[m]\right) \rightarrow \widehat{H F}\left(-S^{3}\right)$. Now, suppose that $n$ is very large. By the adjunction inequality, the map $\widehat{F}_{n, m}$ vanishes for large $m$; moreover, it turns out that its behavior is controlled by the knot invariant $\tau(K)$ :

Proposition 1 OS5, Ra For all sufficiently large $n$, the map $\widehat{F}_{n, m}$ vanishes when $m>\tau(K)$, and is non-trivial when $m<\tau(K)$.

Note that for $m=\tau(K)$ the map $\widehat{F}_{n, m}$ might or might not vanish, depending on the knot $K$.

We'll need two more properties of $\tau(K)$ :

\section{Proposition 2 OS5}

1) If the knot $\bar{K}$ is the mirror image of $K$, then $\tau(\bar{K})=-\tau(K)$.

2) If $K_{1} \# K_{2}$ is the connected sum of two knots $K_{1}$ and $K_{2}$, then $\tau\left(K_{1} \# K_{2}\right)=$ $\tau\left(K_{1}\right)+\tau\left(K_{2}\right)$.

\section{Contact Invariants and Legendrian Knots}

In this section we use properties of the contact invariants to prove Theorem 1 Let the contact manifold $\left(Y_{2}, \xi_{2}\right)$ be obtained from $\left(Y_{1}, \xi_{1}\right)$ by Legendrian surgery, and denote by $W$ the corresponding cobordism. As shown in [LS], the induced map $\widehat{F}_{W}$, obtained by summing over $\operatorname{Spin}^{c}$ structures on $W$, respects the contact invariants; we shall need a slightly stronger statement for the case of Legendrian surgery on $S^{3}$, using the canonical Spin ${ }^{c}$ structure only. 
The canonical $\operatorname{Spin}^{c}$ structure $\mathfrak{k}$ on the Legendrian surgery cobordism $W$ from $S^{3}$ to $S_{-n}^{3}(K)$ (or, equivalently, from $-S_{-n}^{3}(K)$ to $-S^{3}$ ) is induced by the Stein structure and determined by the rotation number of $K$,

$$
\left\langle c_{1}(\mathfrak{k}),[\widehat{\Sigma}]\right\rangle=r,
$$

where $\widehat{\Sigma}$ is the surface obtained by closing up the Seifert surface of $K$ in the attached Stein handle Go. Let $\mathfrak{s}$ be the induced $\operatorname{Spin}^{c}$ structure on $-S_{-n}^{3}(K)$; $\mathfrak{s}$ is the $\operatorname{Spin}^{c}$ structure associated to $\xi$, and $c(\xi) \in \widehat{H F}\left(-S_{-n}^{3}(K), \mathfrak{s}\right)$.

Proposition 3 (cf. [LS] $)$ Let $(W, \mathfrak{k})$ be a cobordism from $\left(S^{3}, \xi_{\text {standard }}\right)$ to $\left(S_{-n}^{3}(K), \xi\right)$ induced by Legendrian surgery on $K$, and let

$$
\widehat{F}_{W, \mathfrak{k}}: \widehat{H F}\left(-S_{-n}^{3}(K), \mathfrak{s}\right) \rightarrow \widehat{H F}\left(-S^{3}\right)
$$

be the associated map. Then

$$
\widehat{F}_{W, \mathfrak{k}}(c(\xi))=c\left(\xi_{\text {standard }}\right) .
$$

Since $c\left(\xi_{\text {standard }}\right)$ is a generator of $\mathbb{Z}=\widehat{H F}\left(S^{3}\right)$, it follows that the map $\widehat{F}_{W, \mathfrak{k}}$ is non-trivial.

Proof of Theorem 1 Since changing the orientation of the knot changes the sign of its rotation number, it suffices to prove the inequality

$$
t b+r \leq 2 \tau(K)-1 .
$$

We may also assume that $t b(K)$ is a large negative number: we can stabilize the knot (adding kinks to its front projection) to decrease the Thurston-Bennequin number and increase the rotation number while keeping $t b+r$ constant.

Writing $-n=t b-1$ for the coefficient for Legendrian surgery and setting $r-n=2 m$, by (1) we can identify the map $\widehat{F}_{W, \mathfrak{k}}$, induced by Legendrian surgery, with $\widehat{F}_{n, m}$ in the notation of Section 2. By Proposition 3, this map does not vanish, so Proposition 1 implies that $m \leq \tau(K)$, which means that

$$
t b(K)+r(K) \leq 2 \tau(K)+1 .
$$

To convert +1 into -1 , we apply this inequality to the knot $K \# K$. Recalling that $t b\left(K_{1} \# K_{2}\right)=t b\left(K_{1}\right)+t b\left(K_{2}\right)+1$ and $r\left(K_{1} \# K_{2}\right)=r\left(K_{1}\right)+r\left(K_{2}\right)$ and using additivity of $\tau$, we get $2 t b(K)+2 r(K)+1 \leq 4 \tau(K)+1$. Then $t b(K)+$ $r(K) \leq 2 \tau(K)$, and (2) now follows, since $t b(K)+r(K)$ is always odd (because the numbers $t b(K)-1=\widehat{\Sigma} \cdot \widehat{\Sigma}$ and $\left\langle c_{1}(\mathfrak{k}),[\widehat{\Sigma}]\right\rangle=r$ have the same parity). 
Example 1 Let $K$ be a $(p, q)$ torus knot. By [OS5], $\tau(K)=\frac{1}{2}(p-1)(q-1)=$ $g(K)$, so Theorem 1 reduces to the Thurston-Bennequin inequality, which is actually sharp in this case. For a $(-p, q)$ torus knot $\bar{K}, \tau(\bar{K})=-\frac{1}{2}(p-$ $1)(q-1)$, and Theorem 10 gives $t b(\bar{K})+|r(\bar{K})| \leq-p q+p+q-2$. Although stronger than the Thurston-Bennequin inequality, this bound is unfortunately not sharp: it follows from the Kauffman and HOMFLY polynomial bounds that $t b(\bar{K})+|r(\bar{K})| \leq-p q$ (and the latter bound is sharp).

\section{An Application: calculating $\tau(K)$}

In this section we use Theorem 1 to determine the invariant $\tau(K)$ for certain knots; essentially, we just give a different proof for some results of OS5] and Li].

Indeed, a Legendrian representative of $K$ and Theorem [1 allows us to find a lower bound for $\tau(K)$. An upper bound is given by the unknotting number of the knot, since $|\tau(K)| \leq g^{*}(K) \leq u(K)$. While $u(K)$ is normally hard to determine, we only need to look at the unknotting number for some diagram of $K$ to find an upper bound for $\tau(K)$.

Example 2 OS5, Li We determine $\tau(K)$ for the knot $K=10_{139}$, shown on Fig. 1 Changing the four crossings circled on the diagram, we obtain an unknot. Therefore, $\tau(K) \leq u(K) \leq 4$, so $\tau(K) \leq 4$. For a lower bound, look

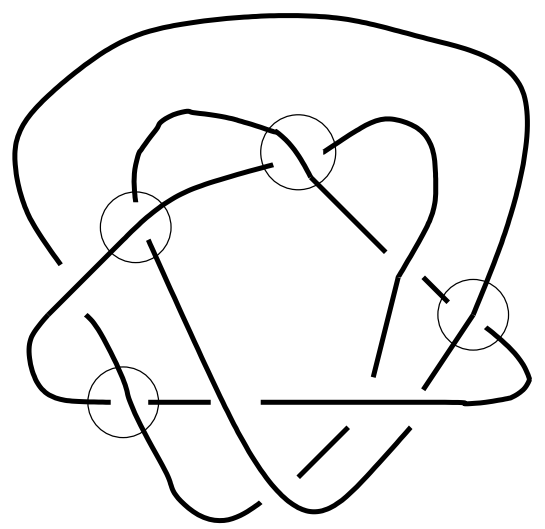

Figure 1: The knot $10_{139}$. Changing the four circled crossings, we obtain an unknot.

at the front projection of the (oriented) Legendrian representative of $10_{139}$ on Fig. 2. The Thurston-Bennequin and the rotation number can be easily found, 


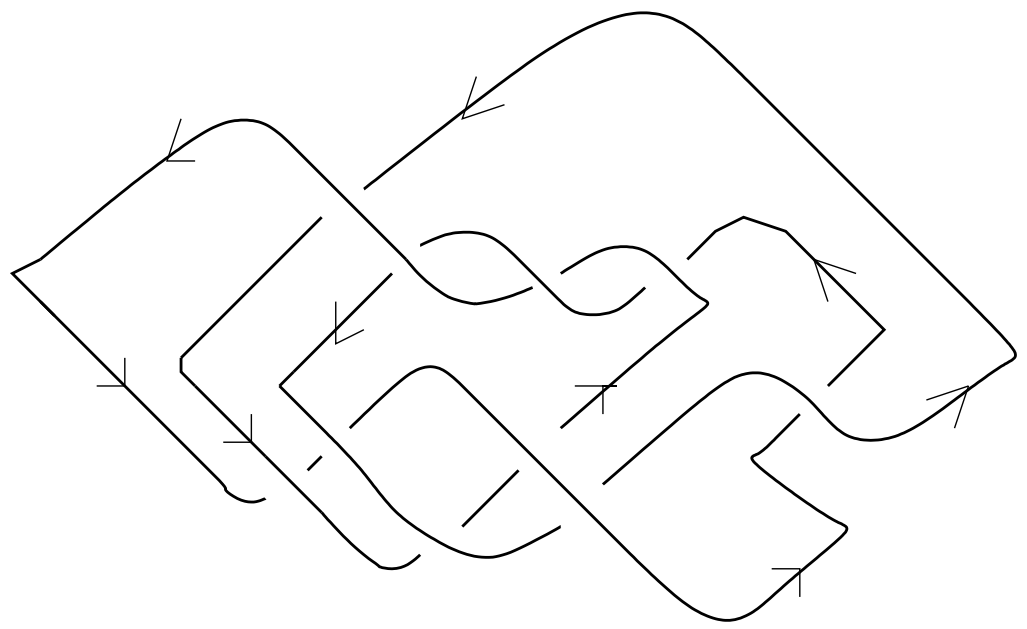

Figure 2: A Legendrian representative of $10_{139}$

since

$$
\begin{aligned}
t b(K) & =\text { writhe }(K)-\# \text { (right cusps) } \\
r(K) & =\#(\text { upward right cusps })-\#(\text { downward left cusps })
\end{aligned}
$$

for an oriented front projection. We compute $t b=6, r=1$, so $2 \tau(K)-1 \geq 7$, and $\tau(K) \geq 4$. It follows that $\tau(K)=4$.

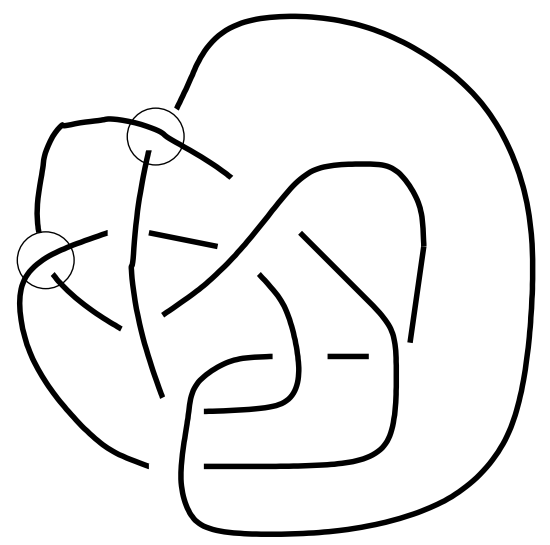

Figure 3: The knot $-10_{145}$. Changing the two circled crossings, we obtain an unknot.

Example 3 Li] Using the same idea, we find $\tau(K)$ for the knot $K=-10_{145}$, shown on Fig. 3] This knot can be unknotted by changing the two circled 
crossings, so $\tau(K) \leq u(K) \leq 2$. On the other hand, for the Legendrian representative shown on Fig. 团 we compute $t b(K)=2, r(K)=1$. Accordingly, $2 \tau(K)-1 \geq 3$; it follows that $\tau(K)=2$.

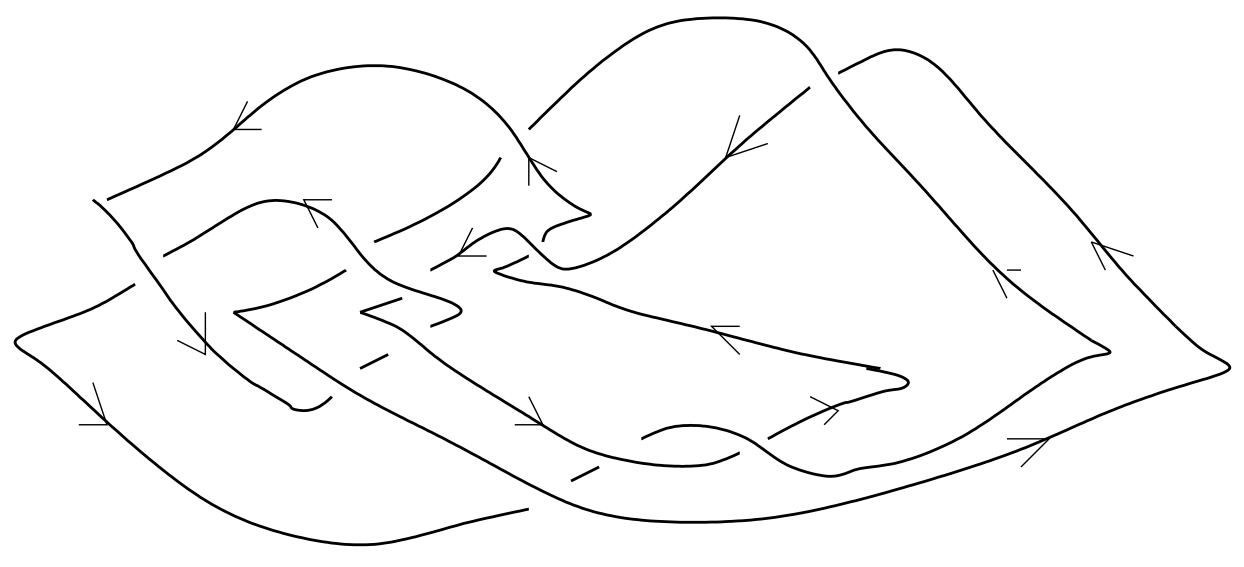

Figure 4: A Legendrian representative of $-10_{145}$

Theorem 2 Li] Let $K$ be a knot which admits a Legendrian representive with positive Thurston-Bennequin number, and let $K_{n}$ be its $n$-th iterated untwisted positive Whitehead double. Then $\tau\left(K_{n}\right)=1$.

We recall that an untwisted positive Whitehead double for a knot $K \subset S^{3}$ is constructed by connecting the knot $K$ and its 0 -push-off $K^{\prime}$ with a cusp; here the 0-push-off is meant to be a copy of $K$, pushed off in the direction normal to a Seifert surface for $K$.

Proof Clearly, for the Whitehead double of any knot, we can obtain an unknot by changing one of the two crossings in the cusp connecting the two copies of the knot. Then the unknotting number for a Whitehead double cannot be greater than one. Now, by a theorem of Akbulut and Matveyev AM the knot $K_{n}$ has a Legendrian representative $L_{n}$ with $t b\left(L_{n}\right)=1$ provided that the original knot $K$ has a Legendrian representive with the positive Thurston-Bennequin number. Since $t b\left(L_{n}\right)+\left|r\left(L_{n}\right)\right| \leq 2 \tau\left(K_{n}\right)-1$, and $\tau\left(K_{n}\right) \leq u(K) \leq 1$, it follows that $\tau\left(K_{n}\right)=1$. 


\section{References}

[AM] S. Akbulut and R. Matveyev, Exotic structures and adjunction inequality, Turkish J. Math. 21 (1997), no. 1, 47-53.

[FT] D. Fuchs and S. Tabachnikov, Invariants of Legendrian and transverse knots in the standard contact space, Topology 36 (1997), no. 5, 1025-1053.

[Go] R. Gompf, Handlebody construction of Stein surfaces, Ann. of Math. (2) 148 (1998), no. 2, 619-693.

[KM] P. Kronheimer and T. Mrowka, Monopoles and contact structures, Invent. Math. 130 (1997), no. 2, 209-255.

[LS] P. Lisca and A. Stipsicz, Heegaard Floer Invariants and Tight Contact ThreeManifolds, arXiv:math.SG/0303280

[Li] C. Livingston, Computations of the Ozsvath-Szabo knot concordance invariant, Geom. Topol. 8 (2004), 735-742.

[OS1] P. Ozsváth and Z. Szabó, Heegaard Floer homologies and contact structures, arXiv:math.SG/0210127

[OS2] P. Ozsváth and Z. Szabó, Holomorphic triangles and invariants for smooth 4manifolds, arXiv:math.SG/0110169.

[OS3] P. Oszváth and Z. Szabó, Holomorphic disks and topological invariants for closed 3-manifolds, arXiv:math.SG/0101206

[OS4] P. Ozsváth and Z. Szabó, Heegaard Floer homology and alternating knots, Geom. Topol. 7 (2003), 225-254.

[OS5] P. Ozsváth and Z. Szabó, Knot Floer homology and the four-ball genus, Geom. Topol. 7 (2003), 615-639.

[Ng] L. Ng, Maximal Thurston-Bennequin number of two-bridge links, Algebr. Geom. Topol. 1 (2001), 427-434.

[Ra] J. Rasmussen, Floer homology and knot complements, Ph.D. Thesis, Harvard, 2003, arXiv:math.GT/0306378

$[\mathrm{Ru}] \mathrm{L}$. Rudolph, The slice genus and the Thurston-Bennequin invariant of a knot, Proc. Amer. Math. Soc. 125 (1997), no. 10, 3049-3050.

[Ta] S. Tabachnikov, Estimates for the Bennequin number of Legendrian links from state models for knot polynomials, Math. Res. Lett. 4 (1997), no. 1, 143-156.

Department of Mathematics, Harvard University

Cambridge, MA 02138, USA

Email: olga@math.harvard.edu

Received: 3 March 2004 